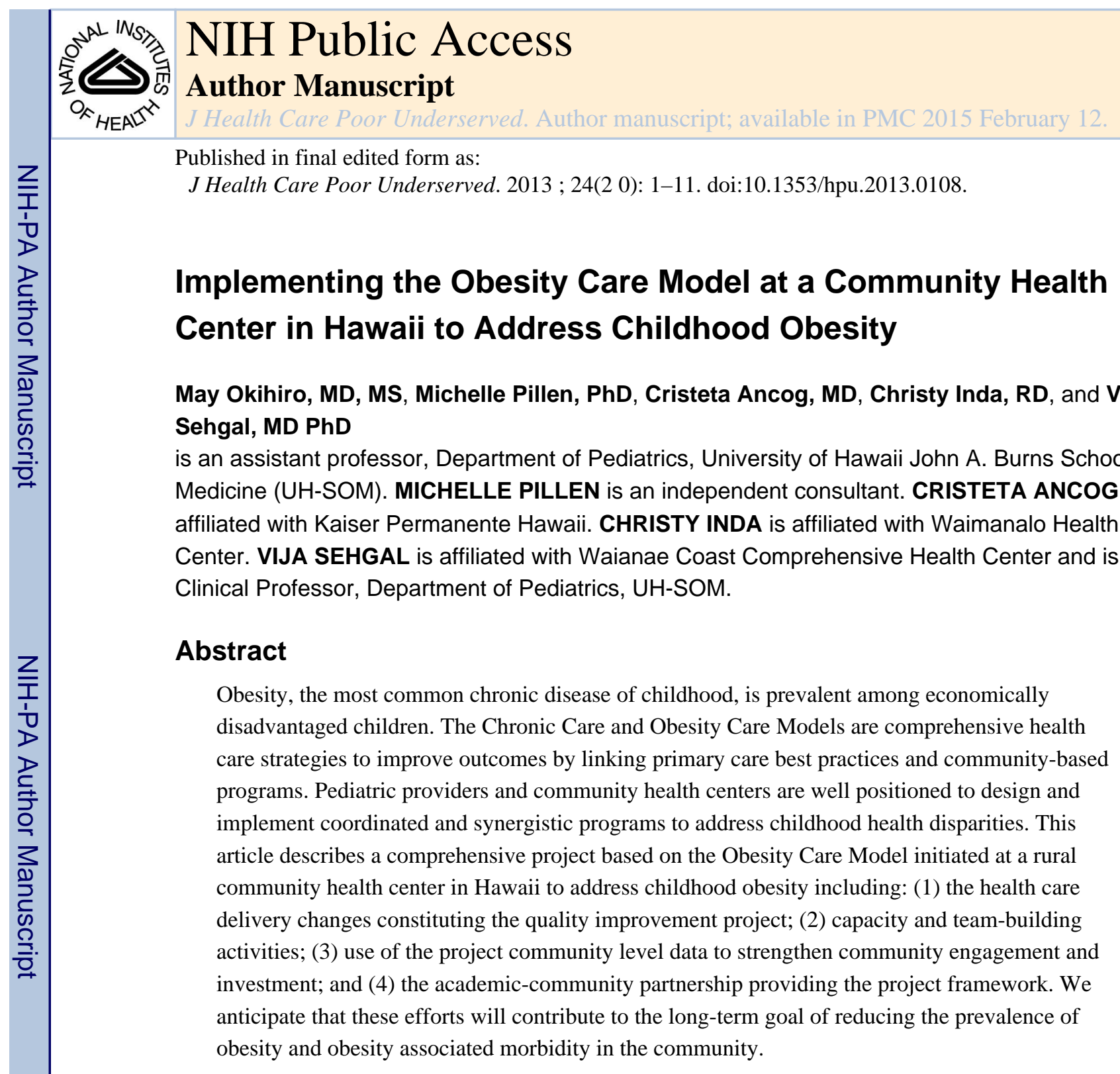

\title{
Keywords
}

Child; obese; health status disparities; quality improvement; Oceanic Ancestry Group; chronic disease; minority

\begin{abstract}
Childhood obesity is the most common chronic disease in childhood and a major risk factor for the development of debilitating disorders such as diabetes and cardiovascular disease. Racial/ethnic minority and economically disadvantaged populations across the nation are at higher risk for obesity than their counterparts. ${ }^{1}$ In Hawaii, Native Hawaiians and other Pacific Islanders have a very high prevalence of obesity, $43.7 \%$ and $59.4 \%$ respectively compared with $20.6 \%$ in Whites. ${ }^{2,3}$
\end{abstract}


The Chronic Care Model (CCM $)^{4,5}$ and its adaptation, the Obesity Care Model (OCM) ${ }^{6}$ are comprehensive models of health care delivery focused on improving outcomes of patients with chronic diseases. The OCM connects primary health care, community-based and public health approaches to support obesity self-management. Thus, the health care system offers individualized assessments and motivates patients and their families to manage weight, while community and public health efforts provide environments to support healthy eating and active lifestyle behaviors (Figure 1). ${ }^{6,7}$

This model offers a comprehensive and sensible approach for communities to manage obesity. Pediatric health care providers are in a key position to understand and manage obesity in children and adolescents under their care. In disadvantaged communities where obesity-related health disparities are the most severe, community health centers (CHC) are well positioned to assist with the development of complementary clinical and public health strategies. To date, however, there is little research documenting the coordinated implementation of such a model, particularly in disadvantaged communities.

Accordingly, a comprehensive project based on the CCM-OCM was initiated at a rural $\mathrm{CHC}$ in Hawaii to address childhood obesity. This consisted of a pediatric primary care quality improvement (QI) research project that integrated clinical flow modifications, new patient education approaches and electronic health record (EHR) enhancements. Recognizing the critical need to reinforce and realize sustainable changes in community behavior to support healthy lifestyles for the children, project data were utilized to inform and motivate $\mathrm{CHC}$ clinicians, CHC administrators, and community leaders as well as to develop new clinic and community-based strategies to address obesity.

This report describes: (1) the community setting; (2) the health care delivery changes and processes that constituted the quality improvement project; (3) capacity and team-building activities among clinicians, academic community physician researchers and community members; (4) use of patient outcome data to strengthen community engagement and investment in addressing childhood obesity; (5) the basis of the academic-community partnership providing the framework for the project; and (6) a brief description of progress to date. The Waianae Coast Comprehensive Health Center (WCCHC) Research Committee, the WCCHC Institutional Review Board, and the University of Hawaii-Committee on Human Subjects, approved the study.

\section{Community Setting}

The project was based at the Waianae Coast Comprehensive Health Center (WCCHC), Hawaii's largest federally qualified community health center ( $\mathrm{CHC}$ ). This $\mathrm{CHC}$ has five satellite offices and provides health care services to the majority of residents of two rural communities on the western side of Oahu, Hawaii's most populated island. These communities are impoverished: per capita income is among the lowest in the State, unemployment is almost double that of the State overall. ${ }^{8}$ In 2011 WCCHC provided health services to 28,775 patients: $52 \%$ were Native Hawaiian, $11 \%$ were Other Pacific Islanders, $15 \%$ were Asian, $42 \%$ were younger than 20 years of age, and $76 \%$ had incomes at or below $200 \%$ of the U.S. federal poverty level. ${ }^{9}$ 


\section{Health Care Delivery Changes}

\section{Clinic flow}

To improve delivery of patient-centered care, clinic flow was redesigned to integrate general pediatric services with pediatric behavioral health and nutrition services. Thus, registered dieticians and the pediatric clinical psychologist were relocated to offices within the clinic. Pediatric clinic leaders developed and implemented a "Healthy Child Weight Policy" to insure that at every clinic visit for pediatric health maintenance (Well Visit), such as Well Child Check Ups and Sports Examinations, body mass index (BMI) is assessed, the parent or teen completes a Wellness Survey (see below) and the child and parent or teen receives counseling on child nutrition and physical activity. A previous $\mathrm{CHC}$ report found that over $50 \%$ of children and adolescents utilizing the clinic were overweight or obese. ${ }^{10}$ Therefore, registered dieticians were integrated into routine Well Visit services for children two years and older, in order to optimize nutrition counseling.

Electronic health record (EHR)—Electronic health record-based enhancements, to assist clinicians and staff in decision-making, were developed to support clinical guidelines advocated by the 2007 Expert Committee on Childhood Obesity. ${ }^{11}$ These included new high-BMI EHR templates that align clinic-based obesity management with best practice guidelines and simplify the documentation of clinical services. The templates also include EHR prompts for laboratory services to screen high-risk patients for diabetes, liver disease and lipid abnormalities and referrals to specialists, such as cardiologists and sleep specialists when appropriate.

Other clinical tools-The Wellness Survey, a 17-question multiple-choice instrument for parents adapted from validated instruments, was developed and implemented to assist CHC pediatricians and dieticians to counsel children and their caregivers. ${ }^{12,13}$ The query topics include fruit, vegetable, milk, and sweetened beverage consumption; breakfast consumption; screen time (time spend utilizing recreational video, computer games and television); physical activity; and sleep. Rice is the grain most commonly eaten by families in Hawaii so a question regarding rice consumption was included in order to assess overconsumption. To assist pediatricians with counseling on child growth and body image, a question on the caregiver's perception of their child's body size was included. This question was derived from a previously validated survey of low-income mothers ${ }^{14}$ and subsequently modified by Young-Hyman ${ }^{15}$ in a study of African American mothers. Caregivers were asked to complete a sentence beginning, "I feel my child is ..." with one of the following response options: "underweight," "a little underweight," "just right," "a little heavy," or "overweight."

Educational handouts from the Hawaii Pediatric Weight Management Toolkit, ${ }^{16}$ a clinician resource developed by a pediatrician and a registered dietician who practice in Hawaii, were field-tested and implemented to guide clinical weight management. To assist clinicians further to counsel children and parents on appropriate growth and lifestyle behaviors, additional educational materials were developed and tested to ensure that families found them useful, locally appropriate, and culturally sensitive. This included a BMI assessment 
handout with an evidence-based color-coded BMI growth chart. ${ }^{17}$ Educational materials were conveniently located at the provider's fingertips in each clinic exam room in order to facilitate accessibility.

\section{Capacity and Team-building}

\section{Quality improvement (QI) team}

Two full-time and five part-time pediatricians and their staff, two registered dieticians, and one pediatric clinical psychologist completed the training described below and actively participated in the implementation of the project. Other members of the team included members of the WCCHC Preventive Health Department, the EHR Department, other pediatricians, and a program evaluator. The project principal investigator (PI), a WCCHC pediatrician and University of Hawaii community physician researcher, led the team.

Training-To promote teamwork and disseminate information on childhood obesity in Hawaii and the new clinic policy and procedures 12 hours of training sessions were held for clinic pediatricians, dieticians, the clinical psychologist and medical assistants. Topics included expert recommendations on the management of childhood obesity, motivational interviewing techniques, community advocacy, cultural aspects of obesity and food in Hawaii, the use of the EHR-based childhood obesity templates, and the Wellness Survey. In addition, proper techniques in measurement of height and weight were reviewed with medical assistants. Clinic staff including receptionists also attended selected trainings sessions.

Team-building-The project used the Plan, Do, Study, Act ${ }^{18}$ framework for continuous quality improvement. This is an iterative process in which problems are identified, changes are planned and then implemented, and data and feedback are used to assess the outcomes and consider modifications. As this was one of the first QI projects at the WCCHC Pediatric Clinic, input from pediatric providers, dieticians, the clinic psychologist, clinic staff, the WCCHC Preventive Health Department, and EHR Departments was sought through a series of meetings and interviews with the project PI and program evaluator throughout the project. The project PI also received regular feedback during her clinical time at the CHC. Concerns and recommendations voiced collectively during these formal and informal interactions were used to modify clinical processes and reassess their impact on clinic flow and on the patients and families.

\section{Community Engagement and Investment}

These preliminary data were disseminated, through a series of meetings and presentations held at the CHC as well as in the community, to WCCHC Preventive Health and Pediatric Staff, WCCHC Administration, members of the WCCHC Board of Directors, the WCCHC Community Research Advisory Committee and other community members. The WCCHC Community Research Advisory Committee is made up of community members and advises the WCCHC Research Committee, as well as individual clinical researchers, in order to foster communication, understanding, investment, and ownership of research priorities at the CHC. 


\section{Community-Academic Partnership}

This project was made possible through a burgeoning $\mathrm{CHC}$-academic partnership and its members' mutual commitment to a community-based research process. It began when the first author (MO), a 13-year veteran community pediatrician employed at WCCHC, was offered a faculty appointment in the Department of Pediatrics of the John A. Burns School of Medicine, University of Hawaii. Over time, the University has provided research resources and infrastructure including training, salary support, mentoring by senior researchers, and research services such as biostatistics, database management, grants development, project coordination, and pilot funds to support new research. Waianae Coast Comprehensive Health Center has a long-standing commitment to research and innovation as a way to improve health care for Waianae Coast residents and address health disparities. It has allowed flexibility in clinical time to participate in rigorous research training, as well as time for research project development and implementation. In addition, the WCCHC has fostered communication between the researcher-clinician and CHC administrators, community research advisors, and community leaders.

\section{Data Collection}

Pediatric providers and staff were interviewed by the program evaluator approximately five months following the project launch and at the end of the project. The interviews were conducted with individuals, pairs, or groups of participants. The focus of the interviews was the successes and challenges encountered in the implementation of the new Healthy Child Weight Policy and integration of the nutritional counseling into pediatric services. The Project PI also met regularly with the program evaluator to share her perspective on the project status.

Body size was categorized by BMI, calculated as weight (in kilograms) divided by height (in meters) squared. BMI was then converted into percentiles for age and sex based on the CDC 2000 growth charts using software available from the CDC. ${ }^{19}$ A child was defined as overweight if BMI was above the 85 th percentile but below the 95th percentile and obese if BMI is at or above the 95th percentile. In addition, selected demographic data of the children was collected from the EHR.

\section{Demographic Characteristics and Baseline Data}

In a 12-month period from September 2010 through August 2011, the research team performed a cross-sectional evaluation of 2,134 unduplicated children ages 2-18 years seen at the WCCHC Pediatric Clinic for Well Visits.

Demographic data are summarized in Table 1. Prevalence of overweight and obesity were very high. Behaviors associated with obesity were common. Obesity-related lifestyle factors, including consumption of large amounts of sugar sweetened beverages and prolonged screen time, were common across all age and BMI groups. In addition, caregivers frequently did not identify their children's weight status accurately; most caregivers of overweight children and adolescents categorized their child's body size as "just right." Strikingly, among children 2-5 years of age, over $80 \%$ of the parents of moderately obese children, and over 
$50 \%$ of parents of severely obese children identified their child's body size as "just right"

(Figure 2).

\section{Progress-To-Date}

\section{Health care delivery}

Pediatric services, including nutrition counseling and behavioral health services, were fully integrated. Dieticians now administer many of the Wellness Surveys to parents at Well Visits in brief encounters that increase their visibility, access, and acceptability. Pediatricians, staff, and parents reported that having the dieticians and psychologists visible and accessible in the pediatric clinic as a key factor in establishing trust and increasing utilization. The registered dieticians and the clinical psychologist now frequently receive "warm hand-offs" from pediatric providers, and direct introductions of more complex patients and his/her parents at the time of their medical appointment.

Using EHR prompts, BMI was assessed at 100\% of Well Visits during the study period. Counseling on appropriate growth, BMI, nutrition and physical activity is now routinely performed at these examinations and easily documented on the enhanced EHR templates. Ordering of appropriate laboratory tests has been streamlined on the EHR templates and is accomplished more consistently for high-risk patients. Colored coded growth charts and educational handouts have been well received and utilized by pediatric providers, staff and families.

Talking about a child's weight and the habits that influence health using the Wellness Survey as the vehicle is the new norm for the clinic. Every child attending a Well Visit, not just the overweight and obese children, is asked about their eating, physical activity, and sleep habits. Pediatricians and dieticians found the Wellness Survey, especially the parents' perception question, useful in their approach to counseling sessions with parents. Prior to this change, talking with children and parents about weight and eating habits was a sensitive topic for pediatric providers, one that was often avoided and because there were fewer resources readily available for patient management.

Capacity and team-building-During the project implementation the collaborative relationship between the WCCHC Pediatric Clinic and Preventive Health providers and staff blossomed. Dieticians altered their schedules to meet the need of pediatricians and their patients for more support in the afternoons, when older children and adolescents are often seen for Well Visits. The psychologist and the dieticians are now regarded as an integral part of the service team, working side-by-side with the pediatric staff. This has helped transform the clinic environment to one in which health and wellness is now also a priority for the clinic staff. Healthier foods are now regular options at clinic celebrations and high calories treats are offered less frequently. Providers and staff regularly share their own challenges and successes in weight loss and maintenance of family health with each other and a growing number now participate, as a group representing WCCHC, in community physical activity events (such as Fun Runs). 
Community engagement and investment-As mentioned, reports of the project's baseline data have been widely disseminated to clinicians, $\mathrm{CHC}$ staff, $\mathrm{CHC}$ administrators, Board members, and other community members. Many from each of the groups found the results "eye-opening," "concerning," motivating and supportive of the CHC's mandate to address health disparities and improve care to the community. Indeed, these groups have contributed to an ongoing discussion of the results and next steps in community action. The results continue to be used to refine Well Visit services and to develop new ideas for research and health services quality-improvement. WCCHC, in collaboration with community and academic partners, is actively developing goals, strategies, and new grants proposals on obesity and diabetes prevention both at the health center and in the community. One recently funded proposal supports the dissemination of this QI project to the WCCHC Satellite Clinics and two other rural CHCs on Oahu.

\section{Discussion}

Community health centers are uniquely positioned to address chronic disease health disparities, such as childhood obesity, through the implementation of best practice guidelines complemented by community-based resources, programs and policies that foster disease self-management and prevention. In this demonstration project, best practice guidelines targeting childhood obesity were implemented using EHR enhancements, a parent survey, pediatric provider and staff training, and a series of cyclical processes to ensure optimal clinic flow, programmatic sustainability, community engagement, cultural appropriateness, and effectiveness.

These community health center resources complement pediatric health care provider efforts to promote healthy growth and development, especially in early childhood. Indeed, the positive influence of pediatric health care providers on parental behavior has been documented in the literature. ${ }^{20,21}$ The use of a Wellness Survey that integrates questions about nutrition, physical activity as well as health and body size perceptions into Well Visits provides a consistent method of assessing family lifestyle behaviors and perceptions in order to provide regular, culturally appropriate feedback and counseling.

The data generated by this study document that this health center population of economically disadvantaged, Native Hawaiian, other Pacific Islander and Asian children has a very high prevalence of obesity, beginning from early childhood. The results of the integrated Wellness Survey, coupled with anthropometric, demographic and diagnostic information collected over time provides a rich, accessible source of data that may be key to achieving the long term goal of reducing the prevalence of obesity and obesity associated morbidity in the community. Over the short and intermediate term, the ongoing data collected serves as the basis for determining the next steps in patient centered health care and clinical quality improvement at the CHC. Community-level data, well-analyzed and reported in a form that is understandable to a lay audience, has the capacity to empower CHC clinicians, community leaders, administrators and researchers to develop new research questions and complementary, locally-relevant community-based strategies to address obesity and chronic disease risk This interaction may result in more effective participatory action research and programming. To be sure, the preliminary data generated from this study 
has reinforced the commitment of the WCCHC Administration and the WCCHC Board of Directors to develop and support sustainable community obesity and diabetes prevention initiatives that target all age groups.

We anticipate that our community and CHC-based efforts in quality improvement, research and community action to address obesity-related health disparities will strengthen further through an evolving relationship between the CHC-based researchers, clinicians, administrators, community members and academic partners. The development of a common vision established by frequent communication, participation and contribution from all stakeholders, and trust made these activities both possible and meaningful.

Every community is unique, with its own strengths, challenges and priorities and thus, the interventions, patient assessments and community engagements that have worked for WCCHC and this community may not be generalizable to other disadvantaged communities and CHCs. However, this CHC EHR-based method that merges clinical and QI research with community action, is lead by CHC-based investigators and community members, and is actively supported by invested academic partners and the CHC administration, is one potentially effective way to address obesity and related chronic conditions in disparate communities in Hawaii.

\section{Acknowledgments}

This work was supported by Kaiser Permanente and the National Institutes of Health, National Institute on Minority Health and Health Disparities Grant No. U54MD007584 and Grant No. P20 MD000173. Its contents are solely the responsibility of the authors and do not necessarily represent the official views of Kaiser Permanente or the NIMHD/NIH. The authors would also like to express their deepest gratitude to Dr. David Easa for his editorial contributions to this paper.

\section{Notes}

1. Eagle TF, Sheetz A, Gurm R, et al. Understanding childhood obesity in America: linkages between household income, community resources, and children's behaviors. Am Heart J. 2012 May; 163(5): 836-43. [PubMed: 22607862]

2. State of Hawaii Department of Health. 2009 State of Hawaii Behavioral Risk Factor Surveillance System. Honolulu, HI: Hawaii State Department of Health; 2013.

3. Mau MK, Sinclair K, Saito EP, et al. Cardiometabolic health disparities in Native Hawaiians and other Pacific Islanders. Epidemiol Rev. 2009; 31:113-29. [PubMed: 19531765]

4. Wagner EH. Meeting the needs of chronically ill people. BMJ. 2001 Oct; 323(7319):945-6. [PubMed: 11679369]

5. Bodenheimer T, Wagner EH, Grumbach K. Improving primary care for patients with chronic illness. JAMA. 2002 Oct 9; 288(14):1775-9. [PubMed: 12365965]

6. Dietz W, Lee J, Wechsler H, et al. Health plans' role in preventing overweight in children and adolescents. Health Aff (Millwood). 2007 Mar-Apr;26(2):430-40. [PubMed: 17339670]

7. Glasgow RE, Orleans CT, Wagner EH. Does the chronic care model serve also as a template for improving prevention? Milbank Q. 2001; 79(4):579-612. iv-v. [PubMed: 11789118]

8. Hawaii State Department of Health, Family Health Services Division. State of Hawaii Primary Care Needs Assessment Data Book 2009. Honolulu, HI: Hawaii State Department of Health; 2010 Jan. Available at: http://hawaii.gov/health/doc/pcna2009databook.pdf

9. Hawai'i Primary Care Association. Community Health Center Profiles. Honolulu, HI: Hawai'i Primary Care Association; Available at: http://www.hawaiipca.net/10/who-are-chcs 
10. Okihiro, M.; Davis, J.; White, L., et al. Body mass index trends in a population of Pacific Island children; Presented at: Pediatric Academic Society Meeting; Washington (DC). May 2005;

11. Barlow SE, Expert Committee. Expert committee recommendations regarding the prevention, assessment, and treatment of child and adolescent overweight and obesity: summary report. Pediatrics. 2007 Dec; 120(Suppl 4):S164-92. [PubMed: 18055651]

12. Brener ND, Kann L, Kinchen SA, et al. Methodology of the youth risk behavior surveillance system. MMWR Recomm Rep. 2004 Sep 24; 53(RR-12):1-13. [PubMed: 15385915]

13. California Department of Public Health. California Children's Healthy Eating and Exercise Practices Survey (CalCHEEPS). Sacramento, CA: California Department of Public Health; 2012 Jan. Available at: http://www.cdph.ca.gov/programs/cpns/Pages/ CaliforniaStatewideSurveys.aspx\#3

14. Baughcum AE, Burklow KA, Deeks CM, et al. Maternal feeding practices and childhood obesity: a focus group study of low-income mothers. Arch Pediatr Adolesc Med. 1998 Oct; 152(10):1010-4. [PubMed: 9790612]

15. Young-Hyman D, Herman LJ, Scott DL, et al. Care giver perception of children's obesity-related health risk: a study of African American families. Obes Res. 2000 May; 8(3):241-8. [PubMed: 10832767]

16. Chock GY, Kerr NA. A Report on the development of the Hawai'i Pediatric Weight Management Toolkit. Hawaii Med J. 2011 Jul; 70(7 Suppl 1):49-51. [PubMed: 21886295]

17. Oettinger MD, Finkle JP, Esserman D, et al. Color-coding improves parental understanding of body mass index charting. Acad Pediatr. 2009 Sep-Oct;9(5):330-8. [PubMed: 19679524]

18. Langley, GL.; Moen, R.; Nolan, KM., et al. The Improvement Guide: A Practical Approach to Enhancing Organizational Performance. 2. San Francisco, CA: Jossey-Bass; 2009.

19. Centers for Disease Control and Prevention. Growth Charts. Atlanta, GA: Centers for Disease Control and Prevention; 2010. Available at: http://www.cdc.gov/growthcharts/

20. Schwartz RP, Hamre R, Dietz WH, et al. Office-based motivational interviewing to prevent childhood obesity: a feasibility study. Arch Pediatr Adolesc Med. 2007 May; 161(5):495-501. [PubMed: 17485627]

21. Taveras EM, Mitchell K, Gortmaker SL. Parental confidence in making overweight-related behavior changes. Pediatrics. 2009 Jul; 124(1):151-8. [PubMed: 19564295] 


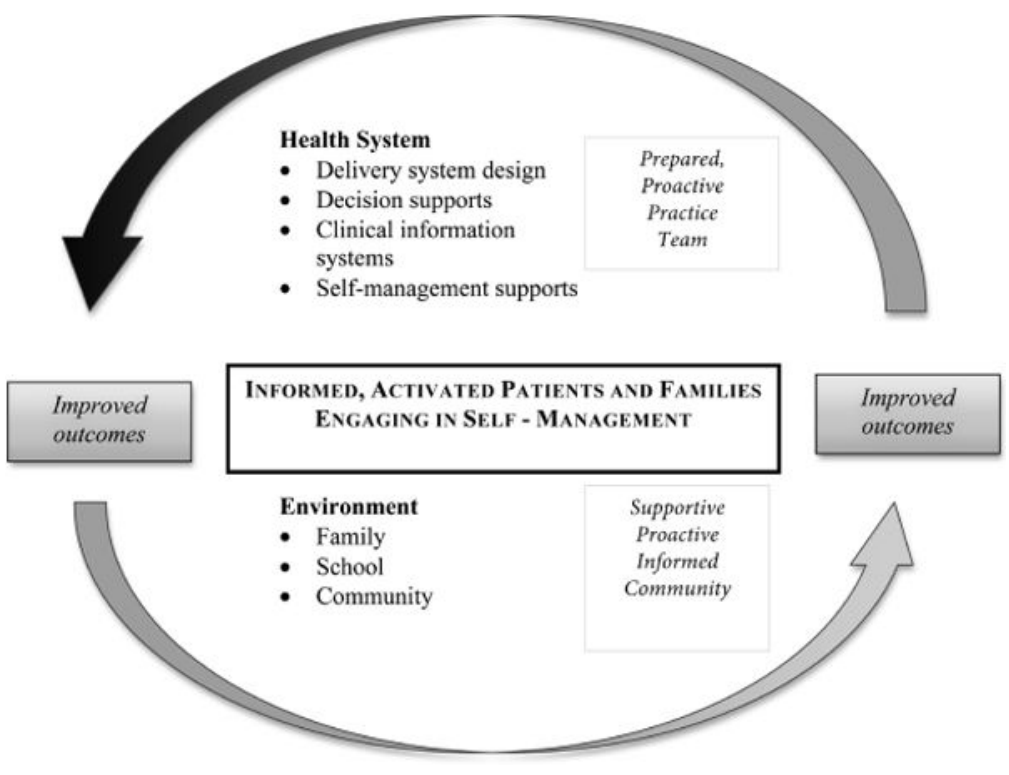

Figure 1. The Obesity Care Model ${ }^{\mathrm{a}}$

aAdapted from Dietz W, Lee J, Wechsler H, et al. Health plans' role in preventing overweight in children and adolescents. Health Aff (Millwood). 2007 Mar-Apr;26(2):430_ 40 and Glasgow RE, Orleans CT, Wagner EH. Does the chronic care model serve also as a template for improving prevention? Milbank Q. 2001;79(4):579-612,iv-v. 


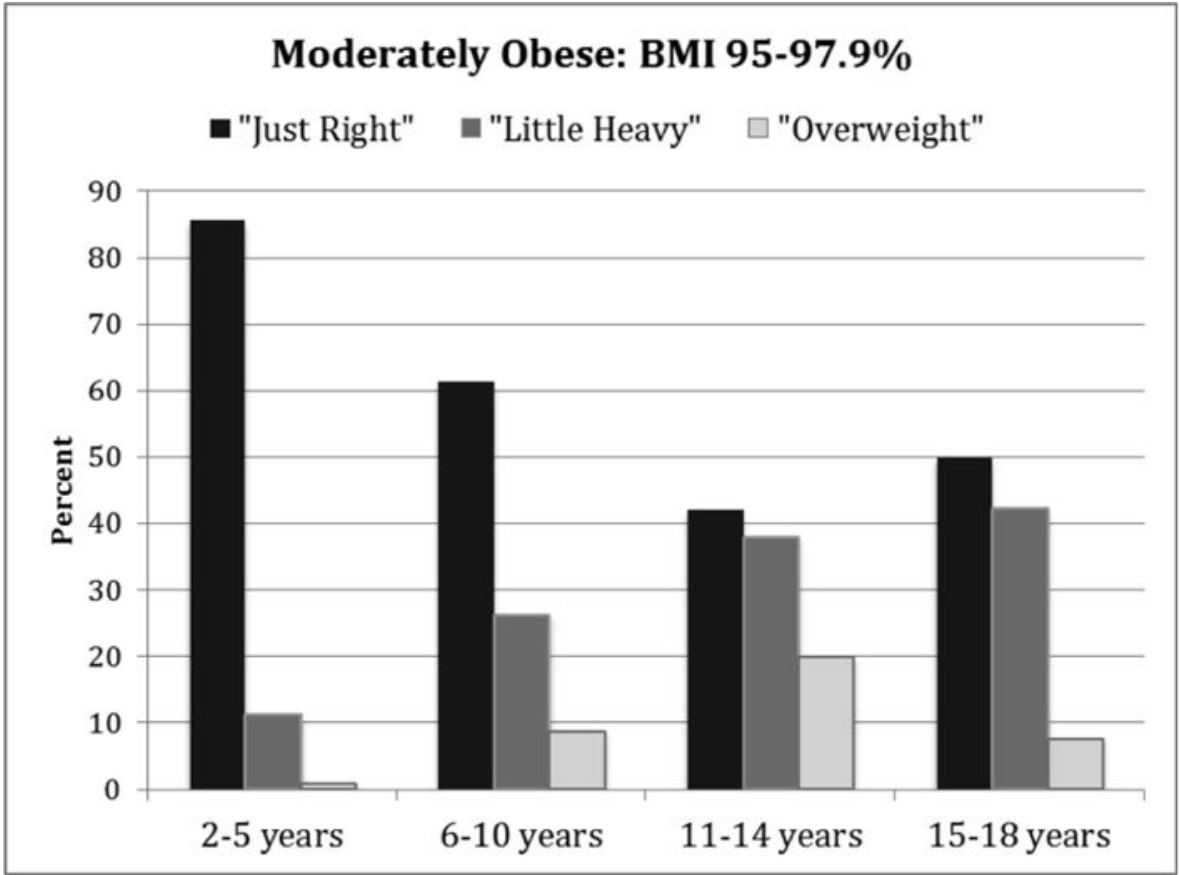

Severely Obese: BMI 98-99.9\%

घ "Just Right" घ "Little Heavy" $\square$ "Overweight"

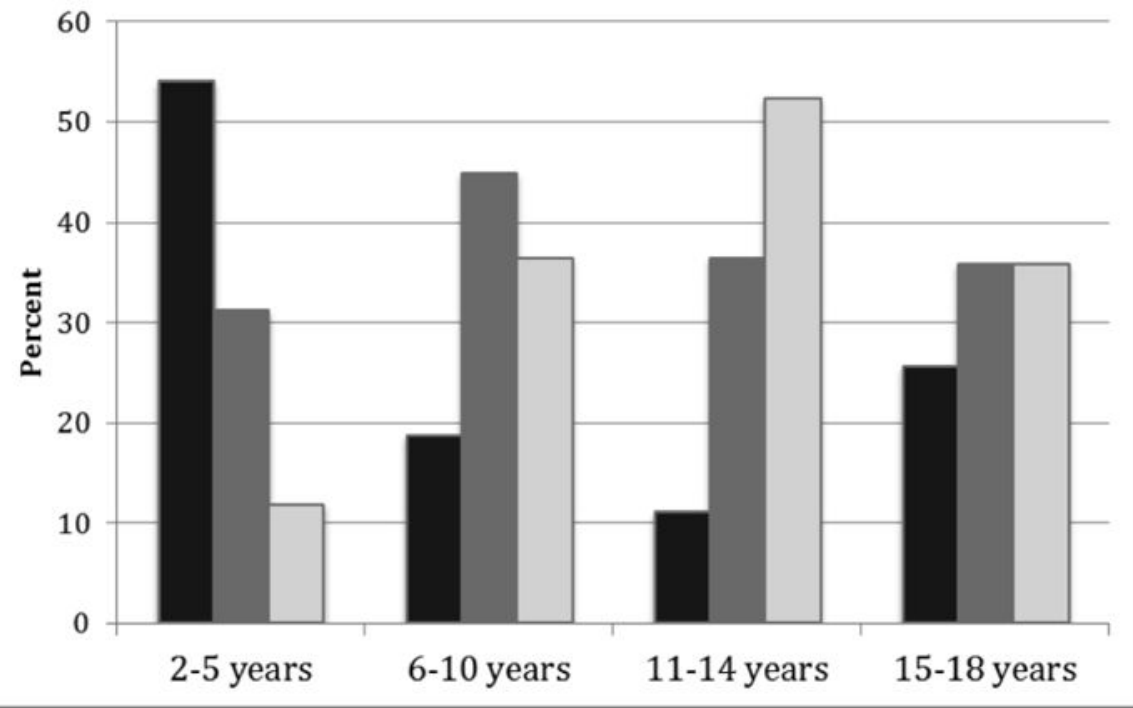

Figure 2.

Parent perception of body size by age and BMI BMI=Body Mass Index 
Table 1

DEMOGRAPHIC DATA, OBESITY PREVALENCE AND LIFESTYLE FREQUENCIES (RESULTS IN PERCENTILES)

\begin{tabular}{lcccc}
\hline Demographics & $\begin{array}{c}\mathbf{2 - 5} \\
\text { years }\end{array}$ & $\begin{array}{c}\mathbf{6 - 1 0} \\
\text { years }\end{array}$ & $\begin{array}{c}\mathbf{1 1 - 1 4} \\
\text { years }\end{array}$ & $\begin{array}{c}\mathbf{1 5 - 1 8} \\
\text { years }\end{array}$ \\
\hline Age groups (N=2,134) & 45 & 26 & 18 & 11 \\
Male gender & 49 & 57 & 57 & 53 \\
Prevalence of overweight and obesity $a$ & & & & \\
Overweight (BMI 85-94.9\%) & 20 & 19 & 24 & 26 \\
Moderately obese (BMI 95-97.9\%) & 10 & 11 & 13 & 12 \\
Severely obese (BMI $\geq 98 \%)$ & 12 & 20 & 17 & 18 \\
Overweight and obesity prevalence combined (BMI $\geq 85 \%)$ & 42 & 50 & 54 & 56 \\
Lifestyle Behaviors & & & & \\
Fast Food ( $\geq 2$ times per week) & 43 & 43 & 43 & 46 \\
Fast Food ( $\geq 4$ times per week) & 5 & 7 & 7 & 11 \\
Rice consumption ( $\geq 2$ servings per dinner) & 19 & 40 & 63 & 71 \\
Sweetened beverages ( $\geq 2$ times per day) & 64 & 59 & 58 & 56 \\
Screen time ( $\geq 3$ hours per day) & 44 & 44 & 51 & 53 \\
\hline
\end{tabular}

${ }^{a}$ Based on US Center for Disease Control 2000 Growth Charts

Centers for Disease Control and Prevention. Growth Charts. Atlanta, GA: Centers for Disease Control and Prevention, 2010. Available at: http:// www.cdc.gov/growthcharts/.

$\mathrm{BMI}=$ Body Mass Index 